\title{
ON CONFORMAL GAUSS MAPS
}

\author{
F.E. BURSTALL
}

\begin{abstract}
We characterise the maps into the space of 2-spheres in $S^{n}$ that are the conformal Gauss maps of conformal immersions of a surface into $S^{n}$. In particular, we give an invariant formulation and efficient proof of a characterisation, due to Dorfmeister-Wang [4,5], of the harmonic maps that are conformal Gauss maps of Willmore surfaces.
\end{abstract}

\section{INTRODUCTION}

A useful tool in conformal surface geometry is the central sphere congruence $[1, \S 67 ; 10]$ or conformal Gauss map [2]. Geometrically, the central sphere congruence of a surface in the conformal $n$-sphere attaches to each point of the surface a 2 -sphere, tangent to the surface at that point and having the same mean curvature vector as the surface at that point. The space of 2 -spheres in $S^{n}$ may be identified with the Grassmannian of $(3,1)$-planes in $\mathbb{R}^{n+1,1}$ and so the central sphere congruence may be viewed as a map, the conformal Gauss map, to this Grassmannian.

The utility of this construction is that it links the (parabolic) conformal geometry of the sphere to the (reductive) pseudo-Riemannian geometry of the Grassmannian. For example, a surface is Willmore if and only if the conformal Gauss map is harmonic $[1, \S 81 ; 2 ; 6 ; 9]$. In another direction, away from umbilic points, the metric induced by the conformal Gauss map, which is in the conformal class of the surface, is invariant by conformal diffeomorphisms of $S^{n}$ and even arbitrary rescalings of the ambient metric $[7,11]$.

The purpose of this short note is to characterise those maps into the Grassmannian which are the conformal Gauss map of a conformal immersion. In so doing, we build on a result of DorfmeisterWang $[4,5]$ which treats the case where the map is harmonic. As a by-product of our analysis, we give an invariant formulation and efficient proof of their result.

It is a pleasure to thank David Calderbank, Udo Hertrich-Jeromin and Franz Pedit for their careful reading of and helpful comments on a previous draft of this paper.

\section{The conformal Gauss map}

We view the conformal $n$-sphere $S^{n}$ as the projective lightcone $\mathbb{P}(\mathcal{L})$ of $\mathbb{R}^{n+1,1}[3$, Livre II, Chapitre VI; 8, Chapter 1]. Here $\mathcal{L}=\left\{v \in \mathbb{R}_{\times}^{n+1,1} \mid(v, v)=0\right\}$ and $($,$) is the signature (n+1,1)$ inner product.

Let $f: \Sigma \rightarrow S^{n}=\mathbb{P}(\mathcal{L})$ be a conformal immersion of a Riemann surface into the conformal $n$-sphere. Equivalently, $f$ is a null line subbundle of the trivial bundle $\underline{\mathbb{R}}^{n+1,1}:=\Sigma \times \mathbb{R}^{n+1,1}$.

Define $f^{1,0} \leq \underline{\mathbb{C}}^{n+2}$ by

$$
f^{1,0}=\operatorname{span}\left\{\sigma, \mathrm{d}_{Z} \sigma \mid \sigma \in \Gamma f, Z \in T^{1.0} \Sigma\right\}
$$

Here the notation $U \leq V$ means $U$ is a subbundle of $V$. That $f$ is a conformal immersion is equivalent to $f^{1,0}$ being a rank 2 isotropic subbundle of $\underline{\mathbb{C}}^{n+2}$. Set $f^{0,1}:=\overline{f^{1,0}}$ and note that ${ }^{1}$ $f^{1,0} \cap f^{0,1}=f$.

The conformal Gauss map of $f$ is the bundle of $(3,1)$-planes $V \leq \underline{\mathbb{R}}^{n+1,1}$ given by

$$
V=\operatorname{span}\left\{\sigma, \mathrm{d}_{Z} \sigma, \mathrm{d}_{\bar{Z}} \sigma, \mathrm{d}_{Z} \mathrm{~d}_{\bar{Z}} \sigma \mid \sigma \in \Gamma f, Z \in \Gamma T^{1,0} \Sigma\right\} .
$$

2010 Mathematics Subject Classification. 53A30 (primary), 53C43 (secondary).

${ }^{1}$ We make no notational distinction between a real bundle and its complexification 
We have a decomposition $\underline{\mathbb{R}}^{n+1,1}=V \oplus V^{\perp}$ which induces a decomposition of the flat connection $\mathrm{d}$ :

$$
\mathrm{d}=\mathcal{D}+\mathcal{N}
$$

where $\mathcal{D}$ is a metric connection preserving $V$ and $V^{\perp}$ while $\mathcal{N}$ is a 1-form taking values in skewendomorphisms of $\underline{\mathbb{R}}^{n+1,1}$ which permute $V$ and $V^{\perp}$.

Remark. We may view $V$ as a map from $\Sigma$ into the Grassmannian of $(3,1)$-planes in $\mathbb{R}^{n+1,1}$ and then $\mathcal{N}$ can be identified with its differential.

The flatness of $\mathrm{d}$ yields the structure equations of the situation:

$$
\begin{aligned}
& 0=R^{\mathcal{D}}+\frac{1}{2}[\mathcal{N} \wedge \mathcal{N}] \\
& 0=\mathrm{d}^{\mathcal{D}} \mathcal{N} .
\end{aligned}
$$

Here $\mathrm{d}^{\mathcal{D}}$ is the exterior derivative on bundle-valued forms with $\mathcal{D}$ used to differentiate coefficients. The conformal Gauss map $V$ is defined by the following properties:

1. $f^{0,1} \leq V$;

2. $f^{0,1} \leq \operatorname{ker} \mathcal{N}^{1,0}$.

Now, for $Z$ a local section of $T^{1,0} \Sigma, \mathcal{N}_{Z}$ is skew while $f^{0,1}$ is maximal isotropic in $V$ so that

$$
\mathcal{N}_{Z} V^{\perp}=\left(\operatorname{ker} \mathcal{N}_{Z \mid V}\right)^{\perp} \subseteq\left(f^{0,1}\right)^{\perp} \cap V=f^{0,1} \leq \operatorname{ker} \mathcal{N}_{Z \mid V}
$$

and we conclude:

Lemma 1.1 (c.f. [4, Proposition 2.2]). If $V$ is the conformal Gauss map of a conformal immersion then $\left(\mathcal{N}^{1,0}\right)^{2}{ }_{\mid V^{\perp}}=0$.

Following [4], we say that $V$ with the property of Lemma 1.1 is strongly conformal.

The conformal Gauss map also satisfies a second order condition. First note that (1.2) tells us that

$$
\mathcal{N}_{Z} V^{\perp} \subseteq f^{0,1}
$$

Moreover, $f^{0,1}$ is $\mathcal{D}^{0,1}$-stable thanks to the following lemma which will see further use in Section 2:

Lemma 1.2. Let $W \leq V$ be maximal isotropic in $V$ with a never-vanishing section $w$ such that $\mathcal{D}_{\bar{Z}} w \in W$, for $\bar{Z} \in T^{0,1} \Sigma$. Then $W$ is $\mathcal{D}^{0,1}$-stable.

Proof. Let $u \in \Gamma W$ be a local section so that $u, w$ locally frame $W$. It suffices to show that $\mathcal{D}_{\bar{Z}} u \in W$. However,

$$
\begin{aligned}
\left(\mathcal{D}_{\bar{Z}} u, w\right) & =-\left(u, \mathcal{D}_{\bar{Z}} w\right)=0 ; \\
\left(\mathcal{D}_{\bar{Z}} u, u\right) & =\frac{1}{2} \bar{Z}(u, u)=0,
\end{aligned}
$$

since $W$ is isotropic. Thus $\mathcal{D}_{\bar{Z}} u \in W^{\perp} \cap V=W$ since $W$ is maximal isotropic in $V$.

In the case at hand, for $\sigma \in \Gamma f$, we have $\mathcal{D}_{\bar{Z}} \sigma \in f^{0,1}$ by definition so Lemma 1.2 applies to show that $f^{0,1}$ is $\mathcal{D}^{0,1}$-stable.

Now contemplate the tension field $\tau_{V}:=* \mathrm{~d}^{\mathcal{D}} * \mathcal{N}$ of $V$. Since $* \mathcal{N}=i\left(\mathcal{N}^{1,0}-\mathcal{N}^{0,1}\right),(1.1 \mathrm{~b})$ yields

$$
\tau_{V}=2 i * \mathrm{~d}^{\mathcal{D}} \mathcal{N}^{1,0}=-2 i * \mathrm{~d}^{\mathcal{D}} \mathcal{N}^{0,1} .
$$

In view of the last paragraph, $\tau_{V} V^{\perp}$ takes values in $f^{0,1}$ since $* \mathrm{~d}^{\mathcal{D}} \mathcal{N}^{1,0} V^{\perp}$ does. However, $\tau_{V}$ is real so that $\tau_{V} V^{\perp}$ takes values in $f^{0,1} \cap f^{1,0}=f$ :

$$
\tau_{V} V^{\perp} \subseteq f .
$$

In particular, since $f$ is a null line subbundle on which $\mathcal{N}$ vanishes, we conclude: 
Proposition 1.3. If $V$ is the conformal Gauss map of a conformal immersion with tension field $\tau_{V}$. Then:

$$
\begin{aligned}
\mathcal{N} \circ \tau_{V \mid V^{\perp}} & =0 \\
\left(\tau_{V}\right)^{2}{ }_{\mid V^{\perp}} & =0 .
\end{aligned}
$$

This line of argument additionally give us some control on the rank of $\mathcal{N}: T \Sigma \otimes V^{\perp} \rightarrow V$ :

Lemma 1.4. Let $V$ be the conformal Gauss map of a conformal immersion $f$, then the set

$$
A:=\left\{p \in \Sigma \mid \mathcal{N}_{Z} V^{\perp}=\mathcal{N}_{\bar{Z}} V^{\perp} \neq\{0\}, Z \in T_{p} \Sigma\right\}
$$

is nowhere dense.

Proof. Any open set in the closure of $A$ must contain an open set where $\mathcal{N}_{Z} V^{\perp}=\mathcal{N}_{\bar{Z}} V^{\perp} \neq\{0\}$. On this latter set, we immediately see from (1.3) that $\mathcal{N}_{Z} V^{\perp}=f$. Since $\tau_{V} V^{\perp} \leq f$ also, by (1.4), we rapidly conclude (c.f. Lemma 2.2 below) that $f$ is $\mathcal{D}^{0,1}$-stable and so $\mathcal{D}$-stable. Since $\mathcal{N} f=0$ also, $f$ is constant: a contradiction.

In the next section, we will establish a generic converse to these results.

\section{RECONSTRUCTION OF $f$ FROM $V$}

Suppose now that we have a bundle $V \leq \underline{\mathbb{R}}^{n+1,1}$ of $(3,1)$-planes and ask whether $V$ is the conformal Gauss map of some conformal immersion $f$. Our task is then to construct $f \leq V$ but, in fact, it will be more convenient to construct $f^{0,1}$ :

Proposition 2.1. Let $W \leq V$ be a maximal isotropic subbundle of $V$ such that:

1. $W$ is $\mathcal{D}^{0,1}$-stable;

2. $\mathcal{N}^{1,0} W=0$, or, equivalently (c.f. (1.2)), $\mathcal{N}_{Z} V^{\perp} \subseteq W$, for all $Z \in T^{1,0} \Sigma$.

Then $f:=W \cap \bar{W}$ is a real, null, line subbundle which, on the open set where it immerses, is conformal with $W=f^{0,1}$ and conformal Gauss map $V$.

Proof. Since $V$ has signature $(3,1), W$ and $\bar{W}$ must intersect in a line bundle, necessarily null and real. Since $f$ is real, $f \leq \operatorname{ker} \mathcal{N}^{1,0} \cap \operatorname{ker} \mathcal{N}^{0,1}$ so that, for $\sigma \in \Gamma f, \bar{Z} \in T^{0,1} \Sigma$,

$$
\mathrm{d}_{\bar{Z}} \sigma=\mathcal{D}_{\bar{Z}} \sigma+\mathcal{N}_{\bar{Z}} \sigma=\mathcal{D}_{\bar{Z}} \sigma \in W,
$$

since $f \leq W$ and $W$ is $\mathcal{D}^{0,1}$-stable. Thus $W=f^{0,1}$ on the set where $f$ immerses. We conclude that, on that set, $f$ is conformal, since $f^{0,1}$ is isotropic and $V$ is the conformal Gauss map of $f$ since $f^{0,1} \leq \operatorname{ker} \mathcal{N}^{1,0}$.

For our main result, we need the following simple observation:

Lemma 2.2. Let $V \leq \mathbb{R}^{n+1,1}$ be a bundle of $(3,1)$-planes with tension field $\tau_{V}$. Let $w=\mathcal{N}_{Z} \nu$, for $\nu \in \Gamma V^{\perp}$ and $Z \in T \Sigma$. Then $\mathcal{D}_{\bar{Z}} w \in \mathcal{N}_{Z} V^{\perp}+\tau_{V} V^{\perp}$.

Proof. For suitable $Z \in T^{1,0} \Sigma, \tau_{V}=\mathcal{D}_{\bar{Z}} \mathcal{N}_{Z}-\mathcal{N}_{[\bar{Z}, Z]}^{1,0}$ so that

$$
\begin{aligned}
\mathcal{D}_{\bar{Z}} w=\mathcal{D}_{\bar{Z}}\left(\mathcal{N}_{Z} \nu\right)=\left(\mathcal{D}_{\bar{Z}} \mathcal{N}_{Z}\right) \nu+\mathcal{N}_{Z}\left(\mathcal{D}_{\bar{Z}} \nu\right) & =\tau_{V} \nu+\mathcal{N}_{[\bar{Z}, Z]}^{1,0} \nu+\mathcal{N}_{Z}\left(\mathcal{D}_{\bar{Z}} \nu\right) \in \mathcal{N}_{Z} V^{\perp}+\tau_{V} V^{\perp} .
\end{aligned}
$$

With all this in hand, we have:

Theorem 2.3. Let $V \leq \underline{\mathbb{R}}^{n+1,1}$ be a bundle of $(3,1)$-planes with tension field $\tau_{V}$. Suppose that:

1. $V$ is strongly conformal.

2. Equations (1.5) hold. 
3. $\left\{p \in \Sigma \mid \mathcal{N}_{Z} V^{\perp}=\mathcal{N}_{\bar{Z}} V^{\perp} \neq\{0\}, Z \in T_{p} \Sigma\right\}$ is empty.

Set $U:=\mathcal{N}_{Z} V^{\perp}+\tau_{V} V^{\perp}$ and restrict attention to the open dense subset of $\Sigma$ where $U$ has fibres of locally constant dimension and so is a vector bundle.

Then $\operatorname{rank} U \leq 2$ and we have:

(a) Where $\operatorname{rank} U=2$, there is a unique real, null line subbundle $f \leq V$ which, where it immerses, is a conformal immersion with conformal Gauss map $V$.

(b) Where $\operatorname{rank} U=1$, there are exactly two real, null line subbundles $f, \hat{f} \leq V$, which, where they immerse, are conformal immersions with conformal Gauss map $V$. In this case, $V$ is harmonic and $f, \hat{f}$ are a dual pair of Willmore, thus $S$-Willmore [6], surfaces.

(c) Where $\operatorname{rank} U=0, V$ is constant and there are infinitely many real, null line subbundles $f \leq V$ defining conformal immersions with conformal Gauss map $V$.

Proof. First note that hypotheses 1 and 2 amount to the assertion that $U \leq V$ is isotropic so that $\operatorname{rank} U \leq 2$.

We now consider each possibility for $\operatorname{rank} U$ in turn.

First suppose that $\operatorname{rank} U=2$. Then $U$ is maximal isotropic in $V$ and is $\mathcal{D}^{0,1}$-stable by Lemma 1.2 in view of Lemma 2.2. By construction $\mathcal{N}_{Z} V^{\perp} \subseteq U$ so that we may take $U=W$ in Proposition 2.1 to learn that $V$ is the conformal Gauss map of $f=U \cap \bar{U}$ where the latter immerses.

Now suppose that $\operatorname{rank} U=1$. We claim that $U=\mathcal{N}_{Z} V^{\perp}$ : first this holds on a dense open set $\Omega$, (if $\mathcal{N}_{Z} V^{\perp}$ vanishes on an open set, so does $\tau_{V}$ ) so that, by hypothesis 3, we have $U \cap \bar{U}=\{0\}$ on $\Omega$. Since $\tau_{V}$ is real, we must have $\tau_{V}=0$ on $\Omega$ and hence everywhere so that the claim follows and $V$ is a harmonic map. It is now immediate that $U$ is $\mathcal{D}^{0,1}$-stable. By hypothesis 3 , we have that $U \cap \bar{U}=\{0\}$ everywhere so that there are exactly two real, null line subbundles $f_{1}, f_{2} \leq V$ orthogonal to $U \oplus \bar{U}$ and we set $W_{i}=f_{i} \oplus U, i=1,2$. Lemma 1.2, applied to a section $w$ of $U$ assures us that each $W_{i}$ is $\mathcal{D}^{0,1}$-stable so that Proposition 2.1 gives that each $f_{i}$ is conformal where it immerses with conformal Gauss map $V$. In this case, the $f_{i}$ are dual Willmore surfaces.

Finally, if $\mathcal{N}^{1,0}=0$ then $\mathcal{N}$ vanishes also so that $V$ is d-stable and so constant. Thus $S^{2}:=\mathbb{P}(\mathcal{L} \cap V)$ is a conformal 2-sphere and any conformal immersion $f: \Sigma \rightarrow S^{2}$ (in particular, any meromorphic function on $\Sigma$, off its branch locus) has $V$ as conformal Gauss map.

Remarks.

1. The caveat that $f$ immerse is not vacuous: one can readily construct $V$ satisfying the hypotheses of Theorem 2.3 for which $f$ we find is constant. Indeed, given constant $f \in \mathbb{P}(\mathcal{L})$, let $W \leq \underline{\mathbb{C}}^{n+2}$ be a non-constant rank 2 isotropic subbundle containing $f$ with $\bar{W}$ holomorphic with respect to the trivial holomorphic structure of $\mathbb{C}^{n+2}$ and choose $V^{\perp}$ to be a complement to $W+\bar{W}$ in $f^{\perp}$. Then it is not difficult to show that $W$ is $\mathcal{D}^{0,1}$-stable and $\mathcal{N}^{1,0} W=\{0\}$.

2. For strongly conformal $V$, equations (1.5) are not independent. Indeed, when rank $\mathcal{N}^{1,0} \mid V^{\perp}=$ 2, $\mathcal{N}_{Z} V^{\perp}$ is maximal isotropic in $V$ so that (1.5a) forces $\tau_{V} V^{\perp} \leq \mathcal{N}_{Z} V^{\perp}$. Thus $\tau_{V} V^{\perp}$ is isotropic and (1.5b) holds. Again, when $\operatorname{rank} \mathcal{N}^{1,0}{ }_{\mid V^{\perp}}=1$, it is easy to see that (1.5a) holds automatically.

In the interesting case of harmonic $V$ (so that $\tau_{V}=0$ ), matters simplify considerably. Here, of course, hypothesis 2 of Theorem 2.3 is vacuous. Moreover, $\mathcal{N}^{1,0}$ is a holomorphic 1-form with respect to the Koszul-Malgrange holomorphic structure of $\underline{\mathbb{C}}^{n+2}$ with $\bar{\partial}$-operator $\mathcal{D}^{0,1}$. It follows that $\mathcal{N}_{Z \mid V^{\perp}}$ has constant rank off a divisor and, moreover, that there is a $\mathcal{D}^{0,1}$-holomorphic subbundle of $\mathbb{C}^{n+2}$ that coincides with $\mathcal{N}_{Z} V^{\perp}$ away from that divisor. In this setting, we conclude with Dorfmester-Wang:

Corollary 2.4 (c.f. [4, Theorem $3.11 ; 5$, Theorem 3.11$]$ ). Let $V \leq \underline{\mathbb{R}}^{n+1,1}$ be a strongly conformal harmonic bundle of $(3,1)$-planes.

Let $U \leq V$ be the $\mathcal{D}^{0,1}$-holomorphic, isotropic bundle that coincides with $\mathcal{N}_{Z} V^{\perp}$ off a divisor. 
(a) if $\operatorname{rank} U=2$, there is a unique real, null line subbundle $f \leq V$ which, where it immerses, is a Willmore, non $S$-Willmore, surface with conformal Gauss map $V$.

(b) if $\operatorname{rank} U=1$ and $U \cap \bar{U}=\{0\}$, there are exactly two real, null line subbundles $f, \hat{f} \leq V$, which, where they immerse, are a dual pair of $S$-Willmore surfaces.

Remark. In the notation of Dorfmeister-Wang [4,5], after a gauge transformation that renders $V, V^{\perp}$ constant, $\mathcal{N}^{1,0}$ is represented by the matrix $B_{1}$.

\section{REFERENCES}

[1] W. Blaschke, Vorlesungen über Differentialgeometrie III, Grundlehren Math., vol. 29, Springer, Berlin, 1929 (German).

[2] R. L. Bryant, A duality theorem for Willmore surfaces, J. Differential Geom. 20 (1984), no. 1, 23-53. MR772125 (86j:58029)

[3] G. Darboux, Leçons sur la théorie générale des surfaces et les applications géométriques du calcul infinitésimal. Première partie, Chelsea Publishing Co., Bronx, N. Y., 1972 (French). MR0396211

[4] J. F. Dorfmeister and P. Wang, Willmore surfaces in spheres via loop groups I: generic cases and some examples (2013), available at arXiv:1301.2756.

[5] - Weierstrass-Kenmotsu representation of Willmore surfaces in spheres (2019), available at arXiv: 1901.08395.

[6] N. Ejiri, Willmore surfaces with a duality in $S^{N}$ (1), Proc. London Math. Soc. (3) 57 (1988), no. 2, 383-416. MR950596

[7] A. Fialkow, Conformal differential geometry of a subspace, Trans. Amer. Math. Soc. 56 (1944), 309-433. MR0011023

[8] U. Hertrich-Jeromin, Introduction to Möbius differential geometry, London Mathematical Society Lecture Note Series, vol. 300, Cambridge University Press, Cambridge, 2003. MR2004958

[9] M. Rigoli, The conformal Gauss map of submanifolds of the Möbius space, Ann. Global Anal. Geom. 5 (1987), no. 2, 97-116. MR944775 (89e:53083)

[10] G. Thomsen, Grundlagen der konformen Flächentheorie, Abh. Math. Sem. Univ. Hamburg 3 (1924), no. 1, 31-56 (German). MR3069418

[11] C. Wang, Moebius geometry of submanifolds in $S^{n}$, Manuscripta Math. 96 (1998), no. 4, 517-534. MR1639852

E-mail address: feb@maths.bath.ac.uk

Dept. of Mathematical Sciences, University of Bath, Bath BA2 7AY, UK. 